\title{
A inserção da Química Verde nos programas de pós-graduação em Química do Brasil: tendências e perspectivas
}

\section{The inclusion of green chemistry in post-graduate programs in Chemistry in Brazil: tendencies and perspectives}

\section{La inserción de la Química Verde en los programas de posgrado en Química en Brasil: tendencias y perspectivas}

Vânia Gomes Zuin, doutora em Ciências e em Educação pela Universidade de São Paulo (USP), professora da Universidade Federal de São Carlos (UFSCAR) e coordenadora brasileira do projeto Sustainable Education and Environmental Development (SEED) in Latin America. Endereço: Rodovia Washington Luís (SP-310), km 235. CEP: 13565-905 - São Carlos, SP. Telefone: (16) 3351-8096. E-mail:vaniaz@ufscar.br.

\section{Resumo}

Este artigo tem como meta investigar as formas pelas quais os princípios da Química Verde têm sido inseridos nos programas de pósgraduação em Química brasileiros. Parte da premissa de que, diante dos atuais desafios que tocam à produção de conhecimentos e formação de recursos humanos voltados à sustentabilidade socioambiental, fazse necessário refletir sobre os temas, as propostas, as potencialidades e os limites relacionados à Química Verde que ocorrem nos cursos ofertados por mais de 40 programas de pós-graduação em Química do País. Observa-se que a quase totalidade desses programas apresenta disciplinas, grupos, linhas e projetos de pesquisa para o desenvolvimento, a análise e a formação que permitem repensar as formas de geração de materiais e processos verdes no contexto acadêmico brasileiro contemporâneo. 
Palavras-chave: Química Verde. Pós-Graduação em Química. Ciência, Tecnologia e Inovação (CT\&I). Sustentabilidade.

\section{Abstract}

This article aims at investigating ways in which the principles of Green Chemistry are included in Brazilian Graduate Programs in Chemistry. Part of the premise is: when facing today's challenges concerning the production of knowledge and the formation of human resources focused on social and environmental sustainability, it has become necessary to reflect on topics, proposals, potentialities and limits related to Green Chemistry, which occur in the courses offered in over 40 Graduate Programs in Chemistry in the country. In almost all of these programs it is possible to observe that they include disciplines, groups, research lines and projects for development, analysis and formation which permit a rethinking of ways of creating green materials and processes in the contemporary Brazilian academic context.

Keywords: Green Chemistry. Graduate Programs in Chemistry. Science, Technology and Innovation (ScT\&I). Sustainability.

\section{Resumen}

Este artículo tiene como objetivo investigar las formas mediante las cuales los principios de la Química Verde se están insertando en los programas brasileños de posgrado en Química. Se supone que, dados los retos actuales que afectan la producción de conocimientos y la formación de recursos humanos centrados en la sostenibilidad socioambiental, es necesario reflexionar sobre los temas, las propuestas, las potencialidades y los límites relacionados a la Química Verde que ocurren en los cursos ofrecidos por más de 40 programas de posgrado en Química de Brasil. Se observa que casi la totalidad de estos programas presenta asignaturas, grupos, líneas y proyectos de investigación para el desenvolvimiento, análisis y la formación que 
permitan repensar las formas de generación de materiales y procesos ecológicos dentro del contexto académico brasileño contemporáneo.

Palabras clave: Química Verde. Posgrado en Química. Ciencia, Tecnología e Innovación (CT\&l). Sostenibilidad.

\section{Introdução}

Nos dias atuais, a construção de caminhos em direção à sustentabilidade é reconhecidamente permeada de obstáculos, muito em função dos riscos existentes, com destaque para os ambientais. Os modelos de desenvolvimento sociossustentável podem ser compreendidos como processos que têm como restrições mais importantes a exploração dos recursos naturais, a orientação científica e tecnológica e os marcos institucionais, com ênfase aos "aspectos qualitativos, notadamente os relacionados com a eqüidade, o uso de recursos - em particular da energia - e a geração de resíduos e contaminantes" (JACOBI, 2003, p. 195; JACOBI; GUNTHER; GIATTI, 2012).

Nesse sentido, durante o século $X X$, pode-se afirmar que a Química mudou significativamente as formas como vivemos, seja devido aos medicamentos, materiais para vestuário, alimentos, à moradia, aos combustiveis para os mais diversos equipamentos, além de uma miríade de outros produtos tidos como essenciais. Entretanto, ainda hoje o processo produtivo - e suas externalidades - é visto também como um empreendimento que pode causar malefícios, haja vista os desastres ambientais ocorridos em todo o mundo, como os de Seveso (Itália, 1976), Bhopal (Índia, 1984) e Toulouse (França, 2001), entre outros (LACANSTER, 2010).

Dadas as demandas contemporâneas em todo o globo, especialmente aquelas relacionadas às questões socioambientais, fazse necessário refletir sobre formas mais adequadas para a produção de conhecimentos científicos e tecnológicos que reduzam ou eliminem a geração de resíduos e efluentes tóxicos. Nesse contexto, insere-se o movimento conhecido como Química Sustentável, benign by design 
ou Química Verde, iniciado com maior ênfase nos anos noventa do século XX, principalmente nos Estados Unidos, na Inglaterra e na Itália. Esse movimento pode ser compreendido como "o uso de uma série de princípios que reduzem ou eliminam a utilização e a geração de substâncias nocivas em seu desenho, manufatura e aplicação" (ANASTAS; WARNER, 1998, p. 11) e objetiva a redução do risco por meio, idealmente, da eliminação do perigo associado às substâncias químicas tóxicas, em vez da restrição de exposição a elas. Dito de outra forma: em vez de se utilizar a abordagem histórica de controlar as concentrações ou emissões de uma substância particular, a Química Verde pretende modificar a natureza intrínseca das substâncias, que seriam então incapazes de causar poluição, tornando desnecessárias as remedições dos impactos ambientais frequentemente observados atualmente (CORREA; ZUIN, 2009; CORREA et al., 2013).

Os 12 princípios propostos por Anastas e Warner (1998) e que balizam as ações no bojo da Química Verde são: 1) prevenção (deve-se prevenir a geração de resíduos em vez de tratá-los); 2) economia de átomos (devem ser planejados métodos sintéticos de modo a maximizar a incorporação de todos os materiais usados no produto final); 3) reações com compostos de menor toxicidade (devem ser planejados métodos que utilizem ou gerem substâncias que possuem pequena ou nula toxicidade); 4) desenvolvimento de compostos seguros (deve-se reduzir ou eliminar a toxicidade sem prejuízo da eficácia); 5) diminuição do uso de solventes e auxiliares (devem ser revistas todas as etapas para a manufatura e o uso de substâncias químicas); 6) eficiência energética (devem ser minimizadas as necessidades energéticas nas transformações químicas); 7) uso de substâncias renováveis (devem ser utilizadas matérias-primas renováveis em detrimento das não renováveis); 8) evitar a formação de derivados (deve ser evitado o uso de reagentes bloqueadores, de proteção ou desproteção, modificadores temporários nos processos químicos e físicos); 9) catálise (devem ser utilizados reagentes catalíticos tão seletivos quanto possível nas transformações químicas); 10) desenvolvimento de substâncias degradáveis (devem ser projetados produtos que cumprem a sua função e não persistem no ambiente, degradando-se em materiais inócuos); 11) análise em tempo real para a prevenção da poluição 
(devem ser estabelecidas metodologias analíticas que possibilitem evitar a formação de substâncias tóxicas em um processo químico); e 12) química segura para a prevenção de acidentes (deve ser planejado o uso de substâncias que minimizem o potencial de explosões, vazamentos, incêndios, etc.).

O movimento da Química Verde tem se ampliado nos últimos anos, envolvendo as principais sociedades de Química mundiais, como a International Union of Pure and Applied Chemistry (IUPAC), que procura estabelecer diretrizes e metas voltadas ao desenvolvimento da Química Verde em todo o mundo (HÖFER, 2009; JESSOP, 2011). No Brasil, a Química Verde tem alcançado maior projeção principalmente no setor industrial, mas também nas universidades e nos demais órgãos governamentais. Desde meados de 2000, várias instituições de ensino, pesquisa, associações profissionais e empresas da área de Química e das Engenharias têm promovido eventos para a difusão da Química Verde. Em 2004, o livro "Green Chemistry in Latin-America” foi publicado como resultado de um projeto apoiado pela IUPAC (TUNDO; ROSSI, 2004).

Em 2007 houve também a criação da Rede Brasileira de Química Verde, que tem como meta atuar institucionalmente de modo a promover inovações científicas e tecnológicas para empresas nacionais, com o apoio da comunidade científica e das agências governamentais, o que incluiu o estabelecimento da Escola Brasileira de Química Verde. Em 2009 e 2010 houve as primeiras publicações de livros voltados à Química Verde com ênfase nos temas, nas pesquisas e potencialidades encontradas no País, caso da obra "Química Verde: fundamentos e aplicações", que recebeu o $52^{\circ}$ prêmio Jabuti de Bronze na categoria "Ciências Exatas, Tecnologia e Informática” da Câmara Brasileira do Livro (CORREA; ZUIN, 2009), e do livro "Química Verde no Brasil: 2010-2030", editado pelo Centro de Gestão e Estudos Estratégicos (CGEE), que apresentou os seguintes temas prioritários: biorrefinarias, pelas rotas termoquímica e bioquímica; alcoolquímica; oleoquímica; sucroquímica; fotoquímica; conversão de CO2; bioprodutos, bioprocessos e biocombustiveis; energias alternativas, que inclui outros eixos transversais como catálise, modelagem e escalonamento de processos (CGEE, 2010). 
Em 2012 o Brasil sediou a Conferência das Nações Unidas sobre Desenvolvimento Sustentável (Rio+20), a partir da qual foi engendrado o documento "O futuro que queremos", que defende, entre várias proposições, o desenvolvimento sustentável para a erradicação da pobreza em todos os níveis. Como parte da programação da Rio+20, o CGEE promoveu nove painéis, sendo um relacionado à discussão da Química Verde e aos desafios para o desenvolvimento sustentável. Aspectos semelhantes relacionados ao aumento de pesquisas que busquem fontes de energia renováveis e processos limpos na área de Química também foram discutidos no livro "Contribuição da pósgraduação brasileira para o desenvolvimento sustentável: Capes na Rio+20”, publicado pela Fundação Coordenação de Aperfeiçoamento de Pessoal de Nivel Superior (Capes), que teve como principal objetivo apresentar os avanços da pós-graduação brasileira na Rio+20 (BRASIL, 2012).

Ainda em 2012, fruto do projeto Sustainable Education and Environmental Development (SEED) in Latin America apoiado pela IUPAC, foi realizada a $4^{\text {th }}$ International IUPAC Conference on Green Chemistry (4 $4^{\text {th }}$ ICGC), em Foz do Iguaçu, Brasil, sob os auspícios da IUPAC e da Sociedade Brasileira de Química (SBQ). A conferência contou com um número superior a 600 participantes provenientes de mais de 45 países, representantes dos setores acadêmico, industrial, governamental e não governamental, que discutiram os recentes avanços e as perspectivas futuras no campo da Química Verde tanto no Brasil quanto no exterior. A programação da $4^{\text {th }}$ ICGC incluiu minicursos, palestras plenárias, palestras técnicas/empresas, apresentações de trabalhos orais e de pôsteres, além de mesas-redondas.

Objetivando aprofundar as discussões nas mesas-redondas da 4th ICGC, algumas questões orientadoras comuns foram enviadas aos seus componentes, caso da mesa intitulada Perspectives on Green Chemistry: the role of the Brazilian research funding agencies, integrada por representantes das principais agências de fomento à pesquisa científica e tecnológica e à formação de pesquisadores do Brasil (como a Capes; o Conselho Nacional de Desenvolvimento Científico e Tecnológico, CNPq; e a Fundação de Amparo à Pesquisa do Estado 
do Rio de Janeiro, Faperj). A fim de verificar os alcances da Química Verde no contexto da pós-graduação em Química do País, as questões orientadoras das discussões realizadas na $4^{\text {th }}$ ICGC foram ampliadas e encaminhadas a todos os coordenadores dos cursos de mestrado profissional, mestrado e doutorado acadêmicos da área de Química do País. Dessa maneira, as respostas apresentadas e debatidas na $4^{\text {th }}$ ICGC proporcionam a elaboração do objetivo deste artigo: compreender a natureza e as formas pelas quais a Química Verde se insere nos programas de pós-graduação em Química do Brasil, além de vislumbrar os principais desafios, potencialidades e limites desse movimento verde na área no contexto acadêmico brasileiro atual.

\section{Delineamento do levantamento e análise dos dados}

Hoje, a área de Química possui 61 programas de pós-graduação recomendados pela Capes, totalizando 96 cursos, sendo 57 cursos de mestrado acadêmico, 37 cursos de doutorado e dois de mestrado profissional, presentes em praticamente todos os estados brasileiros (BRASIL, 2012). Os programas mais antigos encontram-se consolidados e apoiam vários outros cursos, especialmente nas regiões Centro-Oeste e Norte do País, sendo um dos principais desafios da área avançar na consolidação dos cursos nessas regiões. É possivel também observar que os "docentes credenciados na área atuam em todas as subáreas da Química e com uma forte interação com áreas afins (Médicas, Física, Agronomia, Biologia, Biotecnologia, Bioquímica, Materiais, Engenharias, Ensino, etc.). Essa multi- e interdisciplinaridade fica evidenciada na diversidade e qualidade da produção científica” (BRASIL, 2012, p. 106). Além do significativo número de artigos publicados (superior a 10 mil, a maior parte em revistas internacionais), o "avanço tecnológico da área pode ser medido pelo número de patentes depositadas - 272 durante o triênio 2007-2009, correspondendo a um aumento de 55\% em relação ao triênio anterior. Embora a maior parte dos mestres e doutores formados na área de Química" seja absorvida pela academia, cerca de $20-25 \%$ "foram incorporados pelos setores não acadêmicos público e privado. A área está consolidada internacionalmente, sendo uma das áreas que mais cresce em termos de citações/artigos no Brasil" (BRASIL, 2012, p. 106). 
Vários são os temas de pesquisa na área de Química que se relacionam aos princípios da Química Verde:o desenvolvimento de novos materiais, aproveitamento de produtos vegetais, os biocombustiveis (biodiesel e etanol), a produção de hidrogênio, células a combustivel, aditivos e antioxidantes para biocombustíveis, cujo aumento nos últimos cinco anos pode ser explicado em função das demandas e dos incentivos na busca por fontes renováveis e processos verdes. A Faperj lançou o Edital N²7/2012 (Apoio à Pesquisa Científica e Tecnológica em Química Verde - 2012), que teve como objetivo incentivar o desenvolvimento e a inovação de produtos e processos químicos ecologicamente corretos. As diretrizes que balizaram o Edital $N^{\circ}$ 27/2012 basearam-se na obra "Química Verde no Brasil: 2010-2030" (CGEE, 2010), e os projetos aprovados focam majoritariamente síntese orgânica, seguido por biomassa e biocatálise (CORREA et al., 2013). Outras ações indutoras, sem que sejam diretamente denominadas de Química Verde, foram também oportunizadas por outras agências de fomento à pesquisa juntamente com empresas. O programa da Fundação de Amparo à Pesquisa do Estado de São Paulo (Fapesp) voltado à Bioenergia (BIOEN) tem também como meta estimular e articular atividades de pesquisa e desenvolvimento utilizando laboratórios acadêmicos e industriais para promover o avanço do conhecimento e sua aplicação em áreas relacionadas à produção de bioenergia no Brasil.

As demais agências e órgãos, além de incentivar as pesquisas em ciência, tecnologia e inovação nessa direção, têm procurado compreender de que maneira a Química Verde se desenvolve no Brasil. Assim, em função da realização da $4^{\text {th }}$ ICGC, questões formuladas pela sua comissão científica que tinham como meta mapear as formas pelas quais a Química Verde se insere em várias instituições, no Brasil e no mundo, foram encaminhadas aos seus respectivos representantes. Dessa forma, os coordenadores dos programas de pós-graduação em Química do País receberam questões encaminhadas pela coordenação da área de Química da Capes, sob a responsabilidade do prof. Dr. Luiz Carlos Dias, cujos principais resultados foram apresentados na mesaredonda Perspectives on Green Chemistry: the role of the Brazilian 
research funding agencies, da $4^{\text {th }}$ ICGC. As perguntas são listadas a seguir e a análise das respostas é discutida no presente artigo.

1. O tema Química Verde é incluído de alguma forma em seu programa e instituição?

2. Quais são os benefícios e as dificuldades para sua implementação? (Como você avalia a sua execução?)

3. Há propostas para a inserção de disciplinas de graduação ou pós-graduação, seminários, workshops, simpósios?

4. Há propostas para a contratação de docentes com linhas de pesquisa em Química Verde?

5. Quais são as perspectivas futuras ou os planos concretos de sua instituição, a fim de promover a inclusão do tema Química Verde?

6. Comentários que considerar pertinentes, não abordados nas perguntas acima.

Dos 61 coordenadores dos programas de pós-graduação em Química do País, 45 responderam o questionário. Por meio da análise de conteúdo, as respostas foram estudadas, considerando o escrito como ponto de partida para a identificação do conteúdo manifesto (FRANCO, 2007). As categorias estabelecidas se relacionam à ideia principal das perguntas, a saber: institucionalização da Química Verde; potencialidades e limitações da Química Verde; e perspectivas para o estabelecimento e a permanência da Química Verde.

\section{O status quo da Química Verde nos programas de pós-graduação em Química brasileiros}

O conceito de desenvolvimento sustentável se espraia cada vez mais nos programas de pós-graduação brasileiros. Criado em 1980, tal conceito esteve presente na denominada Agenda 21 Global, um documento aprovado por mais de 170 países que participaram da Conferência das Nações Unidas sobre o Meio Ambiente e Desenvolvimento, ocorrida no Rio de Janeiro em 1992, a chamada Rio 92. As principais premissas que compõem esse conceito são as seguintes: 
[...] perspectiva de longo prazo, capacidade de suporte dos ecossistemas, responsabilidade intergerações, princípio da precaução, bem-estar comunitário e participativo, idéias de cooperação, conservação e justiça, bem como concepção de que sustentabilidade comporta várias dimensões, assegurando no mínimo inter-relação da ecológica, econômica e social (BRASIL, 2012, p.11-12).

Essas premissas, que estruturam as bases do conceito de desenvolvimento sustentável, determinaram o engendramento de várias correntes científico- epistemológicas simpáticas a uma postura antipredatória dos chamados recursos naturais. Dentre tais correntes, destaca-se a Química Verde, na área de Química, Engenharias e campos correlatos. Por meio da análise das respostas de coordenadores/ pesquisadores de mais de $73 \%$ dos programas de pós-graduação em Química existentes no País, pode-se observar a forma como a temática da Química Verde amealha cada vez mais espaço tanto nas disciplinas quanto no ethos de tais programas.

Nas respostas à questão "O tema Química Verde é incluído de alguma forma em seu programa e instituição?", 95\% dos entrevistados asseveraram que a temática da Química Verde se encontra organicamente vinculada às disciplinas dos respectivos programas, o que corrobora para a institucionalização desse movimento:

O mesmo tema é abordado na disciplina de Química do Ar, Água e Solo oferecida no programa de pós-graduação (PPGQ5).

No PPGQ temos a disciplina eletiva: "Química Verde e Sustentável". Além disto, o nosso grupo de pesquisa [...] trabalha visando o desenvolvimento de metodologias verdes e a utilização de recursos renováveis em síntese. 0 que permite que os alunos apliquem os princípios da Química Verde ainda na graduação através dos programas de Iniciação Científica (PPGQ2).

Graduação: Curso Bacharelado em Química Ambiental (Noturno). PósGraduação: Disciplina Química Verde, Linhas de Pesquisa "Química Ambiental" e "Química Verde" com ao menos uma dezena de docentes envolvidos. Curso de Inverno: Química Verde com a participação de cerca de 50 alunos de todo país (PPGQ8).

É interessante observar o modo como os docentes e pesquisadores dos programas de pós-graduação em Química 
enfatizaram a presença da Química Verde em suas disciplinas, pesquisas e seus cursos, seja direta ou indiretamente. A presença maciça dos pressupostos da Química Verde necessita ser compreendida no contexto de que a preocupação com as consequências ambientais decorrentes do descarte de resíduos químicos, por exemplo, afeta, muitas vezes de forma irremediável, a vida como um todo. Já na segunda questão feita aos coordenadores/pesquisadores, "Quais são os benefícios e dificuldades para a implementação da Química Verde?", as respostas evidenciaram que muitos consideram evidentes as potencialidades, mas a formação que possibilite o tratamento adequado de temas ligados à Química Verde é hoje um dos principais limitantes para a sua inserção nas instituições:

Os principais benefícios estão na multidisciplinaridade, na grande perspectiva de inovação tecnológica, na atratividade perante a iniciativa privada e órgãos de fomento, na grande visibilidade dos projetos realizados em seu âmbito e na complementaridade de esforços para o desenvolvimento de projetos que realmente poderão fazer a diferença para o futuro da humanidade (PPGQ11).

Os temas relacionados à Química Verde são atuais e despertam grande interesse dos estudantes. Os conhecimentos na área contribuem para uma formação mais ampla e para o desenvolvimento de projetos de pesquisa alinhados com a preocupação com o meio ambiente e com a sustentabilidade. As dificuldades estão mais relacionadas à disponibilidade de professores que atuam em temas centrais da Química Verde em oferecer disciplinas focadas na nesta temática. Em geral, a temática Química Verde está diluída nas disciplinas ministradas em nossos PPG (PPGQ16).

(Os benefícios incluem a) Diminuição da quantidade de resíduos gerados nos laboratórios de graduação e, já há alguns anos, eliminação do uso de reagentes perigosos e poluentes como sais de chumbo e solução sulfocrômica; Coleta seletiva do lixo; Destino final adequado via empresas especializadas em tratamento de resíduos químicos. Dificuldades: Limitações financeiras para dar o devido destino ao lixo comum e aos resíduos dos laboratórios; Formação deficiente dos funcionários responsáveis pelos laboratórios; Conscientização da comunidade em relação à importância do tema; Destinação final de alguns materiais coletados seletivamente, como os vidros dos reagentes (PPGQ20).

Uma vez que (...) não há docentes com formação específica em Química Verde, seria importante uma atualização da temática para os docentes, o que poderia promover uma mudança na atitude e, consequentemente, na formação dos acadêmicos (PPGQ15). 
De forma geral, todos os entrevistados concordaram que as principais benesses à inserção da Química Verde em seus programas se referem à disseminação de uma preocupação formativa, ou seja, ao incentivo do recrudescimento do processo de conscientização da relevância de se refletir sobre o empreendimento científico e os destinos dos resíduos químicos, de tal modo que se desenvolvam resistências quanto a uma perspectiva química instrumentalizada e imediatista (ZUIN, 2011). Trata-se, portanto, do incentivo à presença de uma mentalidade que considera decisiva a história da geração e do descarte de rejeitos, pois se sabe que considerar tal história significa também lidar com a história das relações e produções humanas. Interessante notar que as ênfases atribuídas a tais mentalidades e à formação ilustram a dificuldade de se encontrar profissionais cujas pesquisas estejam direta e explicitamente vinculadas à Química Verde, o que representa mais um reforço à necessidade de que já na graduação os princípios da Química Verde estejam presentes nas respectivas estruturas curriculares. Destaca-se que, dos poucos respondentes que detalharam as maneiras pelas quais a execução dos princípios da Química Verde é avaliada, a procura ou interesse pelas atividades propostas por parte da comunidade acadêmica foi considerada um dos melhores critérios de avaliação.

Na questão sobre as perspectivas futuras ou planos concretos da instituição a fim de promover a inclusão do tema Química Verde, que significa identificar as perspectivas para o estabelecimento e a permanência desse movimento, destacaram-se os seguintes depoimentos:

Os docentes do Departamento de Química já desenvolvem suas pesquisas praticando a Química Verde, tanto nos laboratórios de graduação quanto nos de pesquisa. O principal objetivo é manter o planejamento de modo a não permitir que os novos ingressantes do Departamento, tanto alunos como docentes deixem de praticar os princípios da Química Verde. Neste sentido, Seminários e disciplinas serão programadas para serem ministradas regularmente no Programa (PPGQ25).

A universidade criou em 2012 um grupo chamado (universidade federal) Sustentável, sendo criada uma comissão para discutir e aprimorar as questões relacionadas à Química Verde. O departamento de química possui um representante nessa comissão (PPGQ20). 
Já há grupos de pesquisa atuando em diferentes frentes do envelope "Química Verde". Os concursos para preencher vagas docentes visam captar pesquisadores em várias áreas que o (instituto) considera estratégicas e muitas delas permeiam o tema Química Verde (PPGQ25).

A perspectiva futura será incluir o tema por meio de cursos, workshops e palestras visando não só os alunos, mas também os professores (PPGQ25).

Muitos dos programas de pós-graduação que participaram dessa pesquisa observaram que a presença da Química Verde em suas disciplinas já é uma realidade inconteste. Mas há que se destacar o fato de que tanto as futuras contratações de docentes quanto a contínua preocupação de que os alunos também se interessem pela discussão de seus pressupostos são incentivadas por meio da ampla maioria dos entrevistados. Os comentários adicionais encaminhados pelos entrevistados permitiram compreender outras dimensões que tocam ao objeto deste artigo. Por exemplo, que ações individuais de docentes que participam de outras instituições ou programas de pós-graduação possibilitaram a criação de programas nos quais as pesquisas em Química Verde encontram um lócus específico:

Há um grupo de docentes (...) que está organizando uma Escola Brasileira de Química Verde (EBQV). (...). Criamos uma Rede Brasileira de Química Verde, que tem como uma das finalidades o oferecimento de cursos específicos para estudantes e profissionais da química. O mestrado profissional (...) em Engenharia Petroquímica e de Biocombustíveis, do qual sou docente permanente, já está implementando uma ênfase em QV (Química Verde). No (instituto) acho que não existe uma ação formal, mas há gente daqui envolvida em ações multinstitucionais (PPGQ43).

O fato de as perguntas terem sido encaminhadas a todas as coordenações dos programas de pós-graduação em Química do Brasil, cuja primeira intencionalidade era subsidiar as discussões nas mesas-redondas da $4^{\text {th }}$ ICGC, promoveu reflexões acerca da inserção dos princípios da Química Verde nas suas atividades presentes e futuras, seja na pesquisa, no ensino, na extensão e gestão. Podese asseverar que, em certo sentido, o questionamento também contribuiu para induzir um aprofundamento acerca das motivações, dos temas e das práticas que se relacionam às pesquisas voltadas 
para o desenvolvimento de materiais e procedimentos científicos e tecnológicos menos impactantes ao ambiente no campo da Química:

Neste sentido, as proposições nos dão o direcionamento para requerer junto à administração uma estrutura física e até de cursos de graduação e pós-graduação que prepare os alunos, técnicos e docentes para atuarem na questão ambiental. Ao mesmo tempo o questionário nos alerta para que possamos ampliar nossas ações relativas ao tema da Química Verde no PPGQ (PPGQ13).

Houve relatos a respeito da dificuldade em se definir o significado da Química Verde, que foi enfrentada por meio da solicitação a todo corpo docente para que pensasse sobre os questionamentos colocados:

Desta experiência ficou claro que o tema de fato é muito abrangente. É um tema transversal e muitos de nossos docentes se interessam por ele, talvez até mesmo em decorrência de suas linhas de pesquisas e seus desdobramentos. É importante que muitas das iniciativas de nossos docentes referentes a este tema não são visíveis sob este rótulo "Química Verde". É certo de que são ações espontâneas e motivadas pela percepção e consciência e interesse do docente. 0 tema é relevante e é uma questão de "educação em química”, mas exige conscientização de toda a comunidade para ele. No nosso entender, essa é uma área importante e de futuro, entretanto ela exige cuidados que não costumamos tomar, principalmente quando estamos pressionados pelos resultados (PPGQ35).

Evidencia-se, não apenas nesse depoimento, a demanda por um caráter formativo cultural que, para os entrevistados, urge entrar em todas as instituições acadêmicas. A inserção da Química Verde necessita ir além dos rótulos, já que as demandas atuais e vindouras nos exigem a revisão crítica constante dos objetivos, princípios e das implicações que norteiam a geração de resultados, produtos e processos que se pretendem verdes.

\section{Considerações finais}

A Química Verde se torna cada vez mais presente nos programas de pós-graduação em Química do Brasil. Seja direta ou 
indiretamente, suas premissas conquistam os espaços acadêmicos de tal maneira que se dissemina um ethos afeito à necessidade de que a sociossustentabilidade não se refira apenas a um rótulo que possa ser utilizado na perspectiva somente protocolar de algumas ações, mas sim que seja organicamente assimilada e materializada pelos professores e pesquisadores em Química de nosso País. A discussão sobre os produtos e processos verdes torna-se não só premente, como também decisiva, em tempos em que tanto resíduos quanto seres humanos parecem ser descartáveis, na medida em que não mais são considerados como produtivos. Se a maldição do progresso irrefreável é a regressão irrefreável (ADORNO; HORKHEIMER, 1986), isso não significa que esse seja o destino inexorável dos seres humanos e do ambiente, pois as atuais regressões são construções humanas e, como tais, passiveis de serem modificadas.

Por meio da análise das respostas dos coordenadores/ pesquisadores dos programas de pós-graduação em Química do País, destacou-se fundamentalmente a preocupação com a dimensão educacional/formativa cultural dos docentes e alunos. Seguindo essa linha de raciocínio, há que se enfatizar o fato de que vários dos entrevistados afirmaram que nas contratações futuras de docentes e técnicos de laboratórios os princípios da Química Verde sejam considerados como pontos relevantes nos curricula de ambos.

Definitivamente, tal preocupação não é obra do acaso, pois a conscientização de que os recursos naturais não podem ser predatoriamente utilizados reverbera também na constatação de que os serem humanos que os usam os ressignificam e também precisam deixar de se relacionar predatoriamente. Os pressupostos básicos da Química Verde, na medida em que questionam formas de regressão, referendam a veracidade e a importância central de tal premissa.

Recebido 25/11/2012

Aprovado 30/07/2013

\section{Agradecimentos}

À Capes, ao CNPQ e à Fapesp pelo apoio recebido. 


\section{Referências bibliográficas}

ADORNO, T. W.; HORKHEIMER, M. Dialética do Esclarecimento: fragmentos filosóficos. Tradução de Guido Antonio de Almeida. Rio de Janeiro: Jorge Zahar Editor, 1986.

ANASTAS, P. T.; WARNER, J. C. Green Chemistry: theory and practice. New York: Oxford University Press, 1998.

BRASIL. Ministério da Educação: Coordenação de Aperfeiçoamento de Pessoal de Nivel Superior. Contribuição da pós-graduação brasileira para o desenvolvimento sustentável: Capes na Rio+20. Brasília: Capes, 2012.

CCGE - Centro de Gestão e Estudos Estratégicos. Química verde no Brasil: 2010-2030. Brasília: Centro de Gestão e Estudos Estratégicos, 2010.

CORREA, A. G.; et al. Green Chemistry in Brazil. Pure and Applied Chemistry, v. 85, n. 8, p. 1643-1653, 2013.

CORREA, A. G.; ZUIN, V. G. (Orgs.) Química Verde: fundamentos e aplicações. 1. ed. São Carlos: EDUFSCar, 2009.

FRANCO, M. L. P. B. Análise de Conteúdo. Brasília: Liber, 2007.

HÖFER, R. Sustainable solutions for modern economies. Cambridge: RSC, 2009.

JACOBI, P. R. Educação ambiental, cidadania e sustentabilidade. Cadernos de Pesquisa, v. 118, p. 189-205, 2003.

JACOBI, P. R.; GUNTHER, W. R.; GIATTI, L. L. Agenda 21 e Governança. Estudos Avançados, v. 26, p. 331-340, 2012.

JESSOP, P. Preface to the 3rd International Conference on Green Chemistry (ICGC-3). Pure and Applied Chemistry, v. 83, n. 7, p. 13431343, 2011. 
LACANSTER, M. F. Green Chemistry: an introductory text. Cambridge: RSC, 2010.

TUNDO, P.; ROSSI, R. H. (Eds). Quimica Verde en Latinoamerica. In: Green Chemistry Series. v. 11. Venice: Italy, 2004.

ZUIN, V. G. A inserção da dimensão ambiental na formação de professores de Química. 1. ed. Campinas: Átomo, 2011. 